\title{
Immunostimulatory effects of radioimmunotherapy
}

\author{
Julie Constanzo, ${ }^{1}$ Lorenzo Galluzzi, ${ }^{2,3,4}$ Jean-Pierre Pouget ${ }^{1}$
}

To cite: Constanzo J, Galluzzi L, Pouget J-P. Immunostimulatory effects of radioimmunotherapy. Journal for ImmunoTherapy of Cancer 2022;10:e004403. doi:10.1136/jitc-2021-004403

Accepted 13 January 2022

Check for updates

(C) Author(s) (or their employer(s)) 2022. Re-use permitted under CC BY-NC. No commercial re-use. See rights and permissions. Published by BMJ.

${ }^{1}$ Institut de Recherche en Cancérologie de Montpellier (IRCM), INSERM U1194, Université de Montpellier, Institut régional du Cancer de Montpellier (ICM), Montpellier, France

${ }^{2}$ Department of Radiation Oncology, Weill Cornell Medica College, New York, New York, USA

${ }^{3}$ Sandra and Edward Meyer Cancer Centre, New York, New York, USA

${ }^{4}$ Caryl and Israel Englander Institute for Precision Medicine, New York, New York, USA

Correspondence to Dr Julie Constanzo; julie.constanzo@inserm.fr

\section{ABSTRACT}

Radiation therapy (RT) is known for its ability to kill cancer cells in an immunogenic manner. Recent preclinical data demonstrate that targeted alpha-particle therapy shares with RT the capacity to elicit immunostimulatory effects, standing out as a promising strategy to circumvent immune checkpoint inhibitor resistance in immunologically 'cold' tumors.

Cancer cells exposed to external beam radiation therapy (EBRT) generally undergo immunogenic cell death (ICD), reflecting (1) their intrinsic or EBRT-unmasked antigenicity (ie, the fact that cancer cells expressat baseline or on irradiation-antigenic epitopes that are not covered by central or peripheral tolerance) and (2) the ability of EBRT to drive stress response pathways that ultimately elicit the emission of adjuvantlike signals commonly known as damageassociated molecular patterns (DAMPs). ${ }^{12}$ In a permissive microenvironment, EBRT-driven ICD culminates with the initiation of potent adaptive immune responses that extend systemically, beyond the irradiated lesion (the so-called abscopal response), ${ }^{3}$ and are associated with the emergence of immunological memory. ${ }^{1}$

Of note, while EBRT is suitable for the treatment of localized or oligometastatic tumors, its application to disseminated or diffuse neoplasms is complicated by considerable toxicity to surrounding healthy tissues. At least in part, this obstacle can be circumvented by using targeted radionuclide therapy (TRT), a form of radioimmunotherapy harnessing radiolabeled agents specifically directed to cancer cells. ${ }^{4}$ Indeed, TRT produces macromolecular damage at high density, but only at a short range (for alpha emitters, $<100 \mu \mathrm{m}$ in soft tissues). ${ }^{4}$ These advantages led to the regulatory approval of the alpha emitter ${ }^{223} \mathrm{Ra}$ for use in patients with metastatic, castrationresistant prostate carcinoma, and stimulated the development of several alpha emitterbased radiopharmaceuticals. However, whether TRT resembles EBRT in its capacity to elicit ICD has long remained unclear. Recent data from Lejeune and collaborators demonstrate that TRT with an alpha emitter, an approach that is commonly known as targeted alpha-particle therapy (TAT), elicits transcriptional and molecular signatures of ICD in preclinical tumor models, culminating with the activation of a therapeutically relevant tumor-targeting immune response that (at least in models of colorectal carcinoma) can be further amplified by immune checkpoint inhibitors (ICIs)..$^{5}$

Lejeune and colleagues set to investigate the transcriptional profile of human ovarian carcinoma OVCAR3 cells, which naturally express mesothelin (MSLN), and mouse colorectal carcinoma MC38 cells genetically engineered to express human MSLN (MC38hMSLN cells) on exposure to increasing activities of a MSLN-targeted ${ }^{227}$ Th conjugate (MSLN-TTC). A variety of genes encoding proinflammatory mediators were deregulated in a dose-dependent manner in OVCAR3 and/or MC38-hMSLN cells. Accordingly, OVCAR3 and MC38-hMSLN cells exposed to MSLN-TTC secreted multiple proinflammatory cytokines including interleukin 6 (IL6), IL8, C-C motif chemokine ligand 3 and/ or CCL20, and supernatants from OVCAR3 cells responding to MSLN-TTC efficiently promoted dendritic cell (DC) maturation. Moreover, in line with transcriptomic findings, MSLN-TTC (1) elicited molecular signs of cyclic GMP-AMP synthase (CGAS) signaling in MC38-hMSLN cells (as well as in human mesothelioma NCI-H226 cells) and (2) promoted the emission of multiple ICDrelevant DAMPs in MC38-MSLN, OVCAR3, and NCI-H226 cells. Interestingly, this was associated with the rapid upregulation of interferon alpha and beta receptor subunit 1 (IFNAR1), which is also involved in ICD induction by EBRT, ${ }^{6}$ and CD274 (best known as PD-L1), which instead mediates robust immunosuppressive effects. ${ }^{5}$

In immunocompetent mice MC38-hMSLN tumors, single intravenous injections of MSLN-TTC at increasing activities (ie, 125, 250 , and $500 \mathrm{KBq} / \mathrm{kg}$ ) exhibited considerable activity-dependent efficacy starting at 
$125 \mathrm{KBq} / \mathrm{kg}$. Conversely, some degree of therapeutic efficacy against MC38-hMSLN tumors could only be observed with MSLN-TTC at $500 \mathrm{KBq} / \mathrm{Kg}$ in in $\mathrm{Rag} 2$ ${ }^{-} \mathrm{Il2} \mathrm{rg}^{-/}$mice (which are characterized by a profound lymphoid defect), suggesting that immunological pathways are involved in the mechanism of action (MOA) of TAT. Similar results were obtained on the depletion of $\mathrm{CD}^{+} \mathrm{T}$ cells, formally corroborating the implication of adaptive immunity. In line with this notion, the therapeutic efficacy of MSLN-TTC at $250 \mathrm{KBq} / \mathrm{kg}$ could be increased by PD-L1 blockers, but only when the combinatorial regimen was initiated simultaneously. ${ }^{5}$ These findings lend addition support to the critical important of therapeutic schedule for combinatorial regimens involving immunotherapeutic agents. ${ }^{7}$

Immunohistochemical studies revealed signs of DNA damage in MC38-hMSLN tumors exposed to MSLN-TTC, correlating with (1) repolarization of the $\mathrm{CD}^{+}$infiltrate towards $\mathrm{CD}^{+} \mathrm{T}$ cells expressing coinhibitory receptors including PD-L1; (2) decreased intratumoral abundance of cross-presenting CD103 ${ }^{+}$DCs (but a trend towards higher $\mathrm{CD}_{103^{+}} \mathrm{DC}$ levels in tumor-draining lymph nodes); and (3) accrued tumor infiltration by immunosuppressive M2-like macrophages. These findings suggest that MSLN-TTC elicits at least some degree of tumor-targeting immunity coupled to the activation of compensatory immunosuppressive pathways commonly associated with effector interferon gamma signaling. Further demonstrating the implication of adaptive immunity in the MOA of TAT, immunocompetent mice eradicating MC38-hMSLN tumors on MSLN-TTC therapy were completely protected from the inoculation of an otherwise tumorigenic dose of MC38-hMSLN cells. ${ }^{5}$
Taken together, the results by Lejeune and colleagues add to previous studies demonstrating that TRT mediates immunostimulatory effects ${ }^{589}$ as they identify the ability of TAT to elicit ICD-associated signal transduction pathways (figure 1). Whether TRT elicits bona fide ICD (as assessed in gold standard vaccination assays), however, remains to be clarified. Importantly, the ability of EBRT to elicit immunostimulatory effects downstream of CGAS activation by mitochondrial DNA (mtDNA) is highly sensitive to dose per fraction, at least in part reflecting the dose-dependent upregulation of the exonuclease threeprime repair exonuclease 1 (TREX1) ${ }^{6}{ }^{10}$ Specifically, multiple mouse and human cancer cells appear to exhibit a dose threshold for TREX1 upregulation at 10-12Gy, resulting in compromised type I interferon (IFN) secretion as compared with doses $<8-10 \mathrm{~Gy} .{ }^{10}$ Extrapolation of these data to TRT is not straightforward because TRT is delivered at much lower dose rates than EBRT. Moreover, irradiation by TRT persists until the radionuclide is excreted or it decays, leading to protracted dose accumulation over time. Thus, the biological effects of EBRT and TRT are largely different from each even when dose is comparable. Finally, clinical TRT is generally planned based on activity $(\mathrm{KBq} / \mathrm{mL}$ or $\mathrm{KBq} / \mathrm{kg}$ ) rather than dose, even though the former does not directly correlate with therapeutic outcome as dose delivered by a specific activity depends on a variety of factors including pharmacodynamic and pharmacokinetic parameters. In line with this notion, TREX1 was upregulated in MC38-hMSLN cells exposed to MSLN-TTC at $50 \mathrm{KBq} / \mathrm{mL}$ in vitro, but not to $250 \mathrm{KBq} / \mathrm{Kg}$ in vivo, ${ }^{5}$ suggesting that the dose threshold was not reached in the latter setting. Thus, accurate dosimetry stands out as a critical tool to deconvolute the
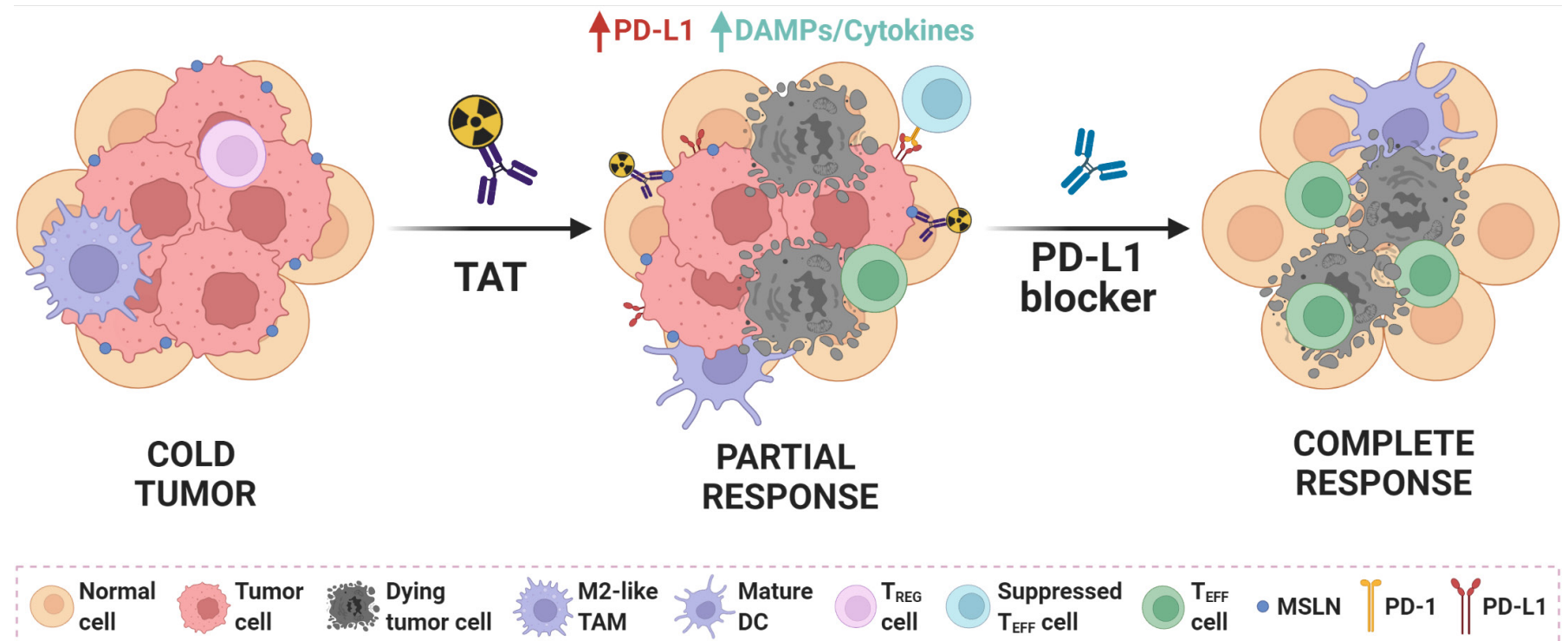

Figure 1 Immunostimulation by TAT. Immunologically cold tumors exhibiting robust infiltration by regulatory $T$ ( $T_{\text {REG }}$ ) cells and M2-like tumor-associated macrophages (TAMs), but limited amounts of mature dendritic cells (DCs) and effector T ( $\left.T_{E F F}\right)$ cells, are generally insensitive to immune checkpoin inhibitors (ICls). At least in preclinical tumor models, targeted alpha-particle therapy (TAT) has been successfully harnessed to inflame the microenvironment of immunologically cold tumors in support of restored ICl sensitivity, pointing to TAT as to a promising combinatorial partner for ICl-based immunotherapy in the clinical setting. DAMP, damage-associated molecular pattern. 
radiobiology or TAT and propose rational approaches towards clinical TAT applications.

Irrespective of this and other obstacles, TRT represents a promising tool to convert immunologically 'cold' tumors into inflamed lesions that respond to ICIs. It will be interesting to see whether this therapeutic paradigm will translate into a clinical reality. A few clinical trials testing this possibility are currently ongoing.

Acknowledgements This work was supported by SIRIC Montpellier Cancer Grant INCa_Inserm_DGOS_12553, INCa-Cancéropôle GSO, AVIESAN PCSI (\#ASC20025FSA), LABEX Mablmprove, and Fondation ARC pour la Recherche sur le Cancer (\#ARCPJA32020060002266). The LG lab is supported by a Breakthrough Level 2 grant from the US DoD BRCP (\#BC180476P1), by the 2019 Laura Ziskin Prize in Translational Research (\#ZP-6177, PI: Formenti) from the Stand Up to Cancer (SU2C), by a Mantle Cell Lymphoma Research Initiative (MCL-RI, PI: ChenKiang) grant from the Leukemia and Lymphoma Society (LLS), by a startup grant from the Dept. of Radiation Oncology at Weill Cornell Medicine (New York, US), by a Rapid Response Grant from the Functional Genomics Initiative (New York, US), by industrial collaborations with Lytix Biopharma (Oslo, Norway) and Phosplatin (New York, US), and by donations from Phosplatin (New York, US), the Luke Heller TECPR2 Foundation (Boston, US), Sotio a.s. (Prague, Czech Republic), Onxeo (Paris, France), Ricerchiamo (Brescia, Italy), and Noxopharm (Chatswood, Australia).

Contributors The commentary results from the discussion and the consensus of all authors listed (JC, LG, and J-PP).

Funding The authors have not declared a specific grant for this research from any funding agency in the public, commercial or not-for-profit sectors.

Competing interests $L G$ has been holding research contracts with Lytix Biopharma and Phosplatin, and has received consulting/advisory honoraria from Boehringer Ingelheim, AstraZeneca, OmniSEQ, Onxeo, The Longevity Labs, Inzen, and the Luke Heller TECPR2 Foundation. All other authors have no conflicts to declare.

Patient consent for publication Not applicable.

Ethics approval This study does not involve human participants.
Provenance and peer review Not commissioned; externally peer reviewed.

Open access This is an open access article distributed in accordance with the Creative Commons Attribution Non Commercial (CC BY-NC 4.0) license, which permits others to distribute, remix, adapt, build upon this work non-commercially, and license their derivative works on different terms, provided the original work is properly cited, appropriate credit is given, any changes made indicated, and the use is non-commercial. See http://creativecommons.org/licenses/by-nc/4.0/.

\section{REFERENCES}

1 Rodriguez-Ruiz ME, Rodriguez I, Leaman O, et al. Immune mechanisms mediating abscopal effects in radioimmunotherapy. Pharmacol Ther 2019;196:195-203.

2 Wu Chuang A, Kepp O, Kroemer G, et al. Endoplasmic reticulum stress in the cellular release of damage-associated molecular patterns. Int Rev Cell Mol Biol 2020;350:1-28.

3 Ngwa W, Irabor OC, Schoenfeld JD, et al. Using immunotherapy to boost the abscopal effect. Nat Rev Cancer 2018;18:313-22.

4 Pouget J-P, Constanzo J. Revisiting the radiobiology of targeted alpha therapy. Front Med 2021;8:692436.

5 Lejeune P, Cruciani V, Berg-Larsen A, et al. Immunostimulatory effects of targeted thorium-227 conjugates as single agent and in combination with anti-PD-L1 therapy. J Immunother Cancer 2021;9:e002387.

6 Yamazaki T, Kirchmair A, Sato A, et al. Mitochondrial DNA drives abscopal responses to radiation that are inhibited by autophagy. Nat Immunol 2020;21:1160-71.

7 Petroni G, Galluzzi L. Impact of treatment schedule on the efficacy of cytostatic and immunostimulatory agents. Oncoimmunology 2021;10:1889101.

8 Patel RB, Hernandez R, Carlson P, et al. Low-dose targeted radionuclide therapy renders immunologically cold tumors responsive to immune checkpoint blockade. Sci Transl Med 2021;13:eabb3631.

9 Li M, Liu D, Lee D, et al. Targeted alpha-particle radiotherapy and immune checkpoint inhibitors induces cooperative inhibition on tumor growth of malignant melanoma. Cancers 2021;13:3676.

10 Vanpouille-Box C, Alard A, Aryankalayil MJ, et al. DNA exonuclease TREX1 regulates radiotherapy-induced tumour immunogenicity. Nat Commun 2017;8:15618. 